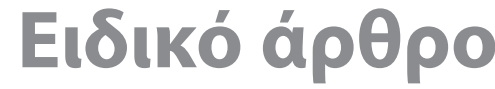 Special article
}

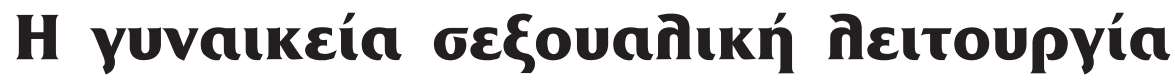

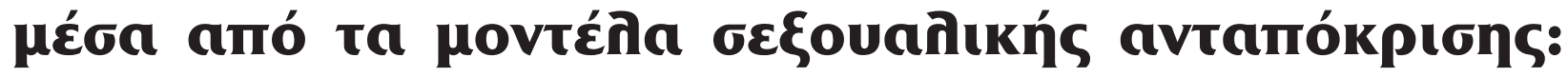 Aпró то DSM-IV бто DSM-5}

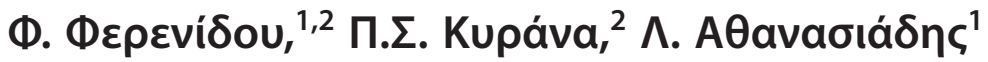

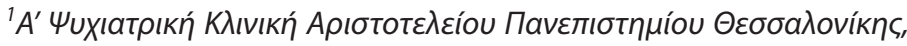

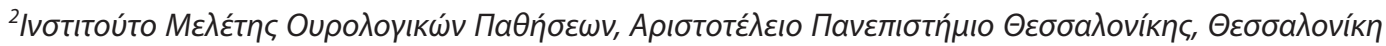

Uuxıатріkń 2017, 28:322-331

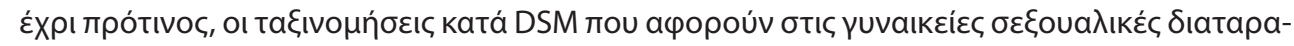

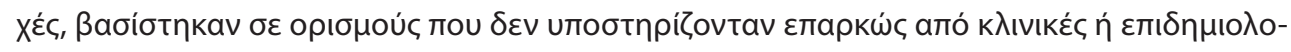

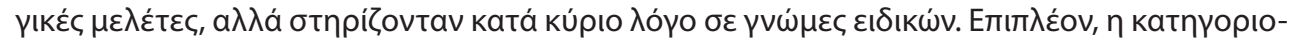

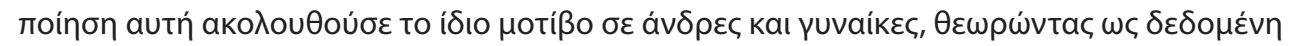

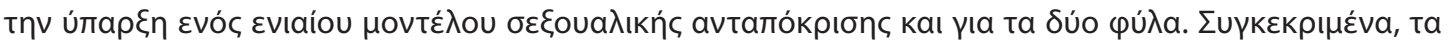

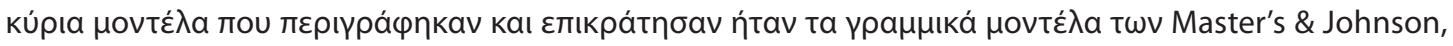

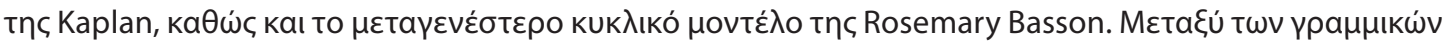

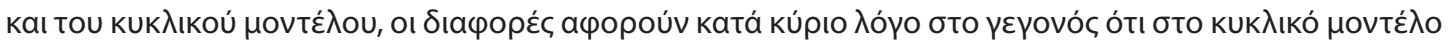

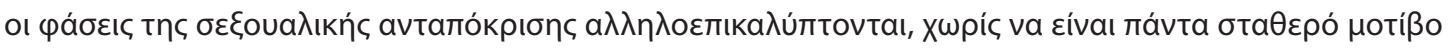

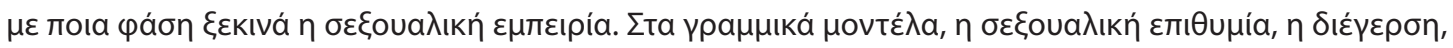

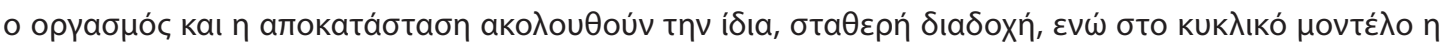

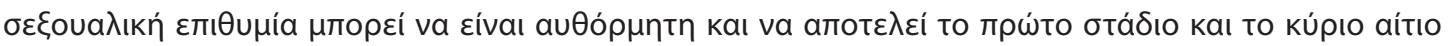

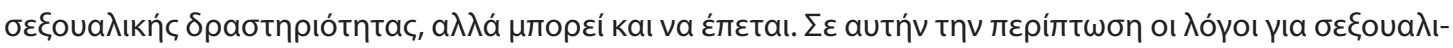

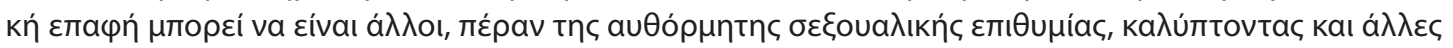

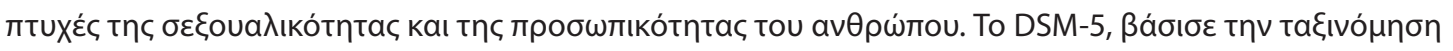

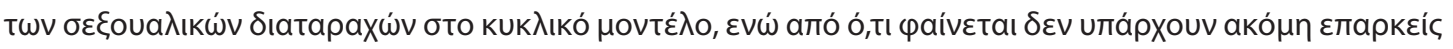

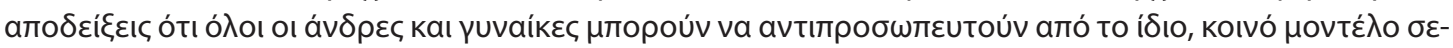

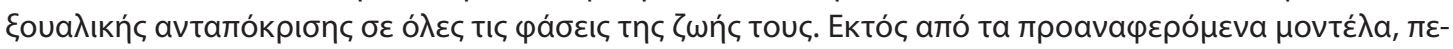

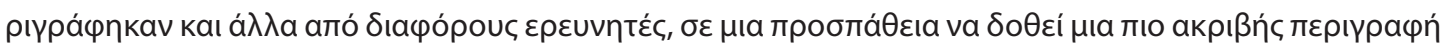

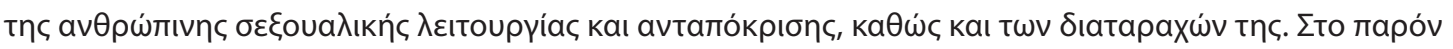

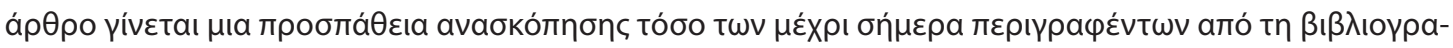

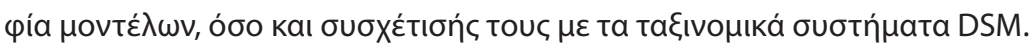

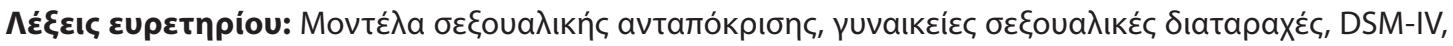
DSM-5. 


\section{Eıбaywyń-

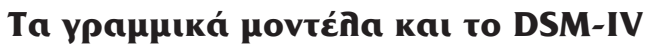

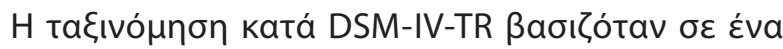

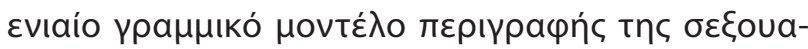

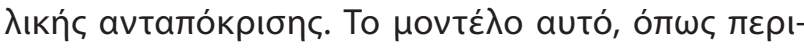

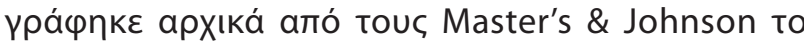

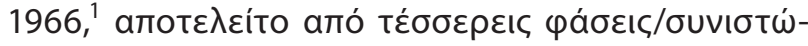

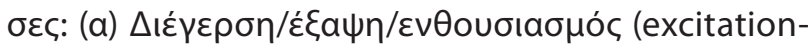

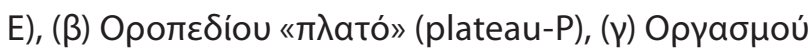
(orgasm-O), (ठ) Апокатáбtaons (resolution-R). To

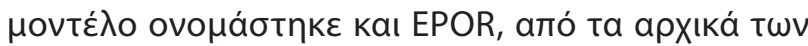

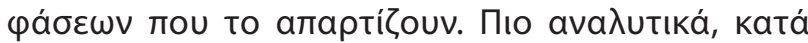

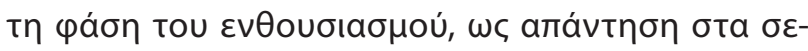

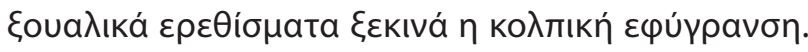

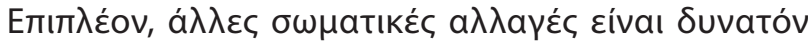

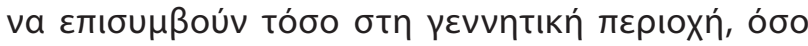

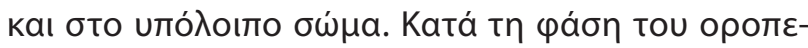

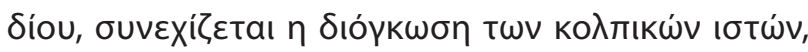

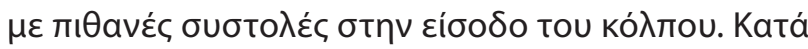

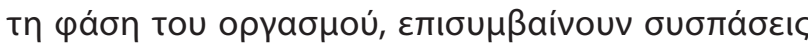

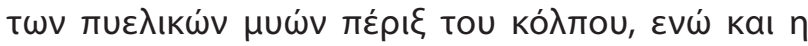

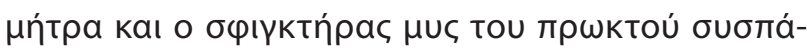

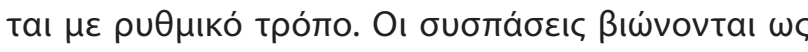

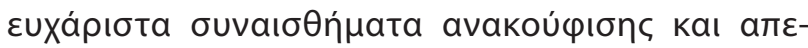
$\lambda \varepsilon \cup \theta \varepsilon ́ \rho \omega \sigma n \varsigma . ~ \Sigma u ́ \mu \varphi \omega v a ~ \mu \varepsilon$ touৎ Masters \& Johnson,

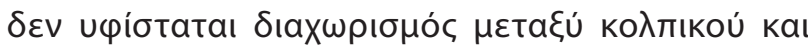

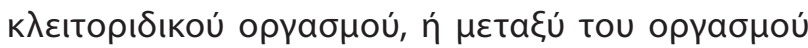

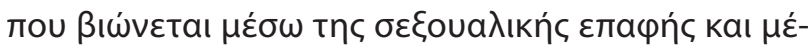

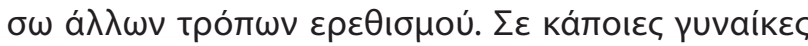

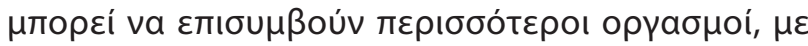

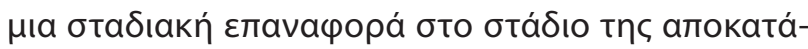

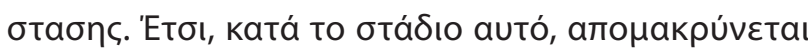

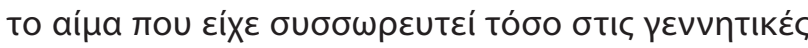

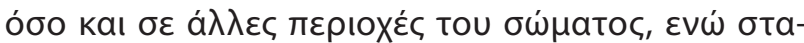

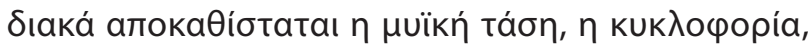

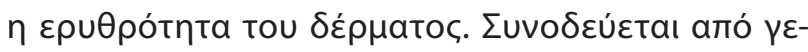

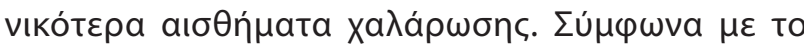

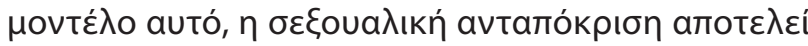

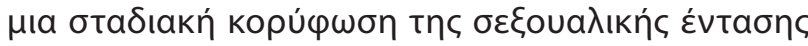
$\mu \varepsilon \dot{x \rho ı ~ т \eta v ~ \varepsilon \pi \varepsilon ́ \lambda \varepsilon \cup \sigma \eta ~ t o u ~ o p ү a \sigma \mu o u ́ . ~}$

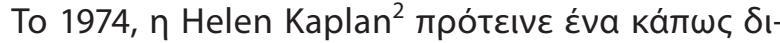

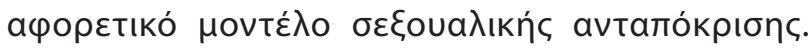

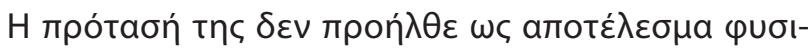

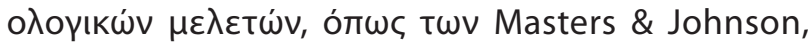

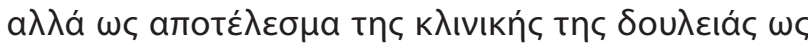

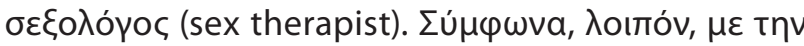

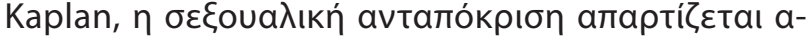

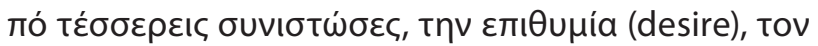

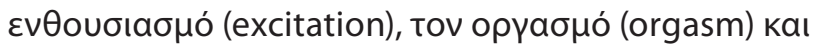

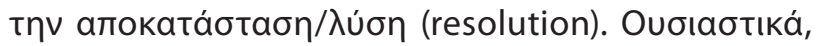

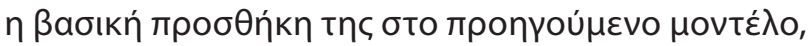

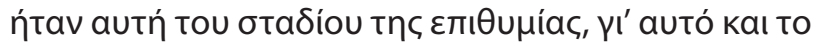

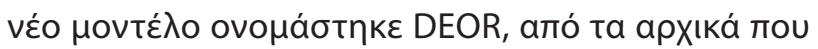

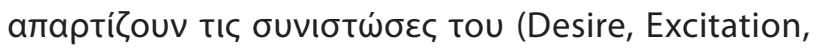

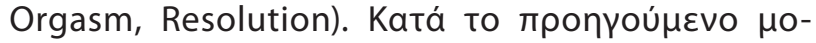

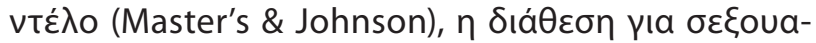

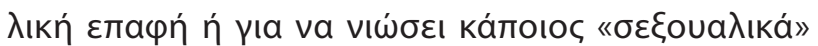

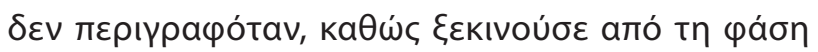

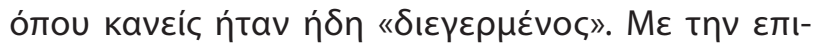

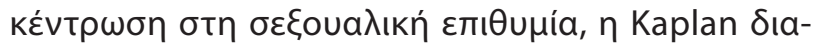

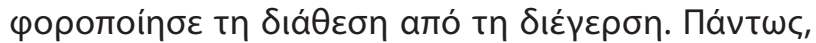

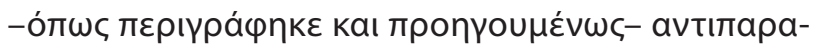

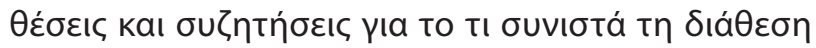

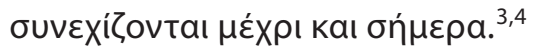

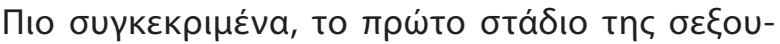

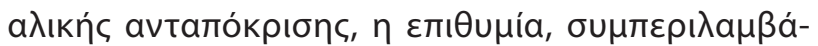

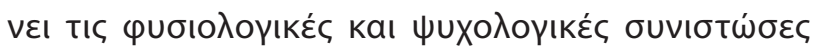

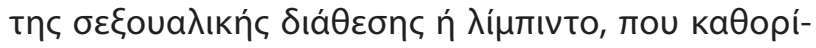

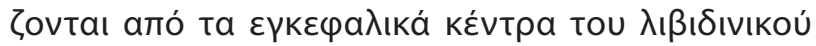
(

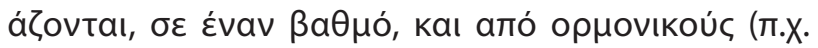

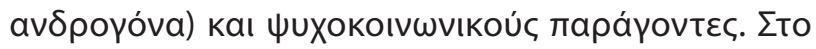

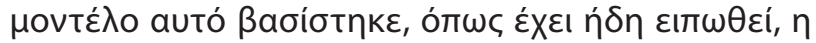

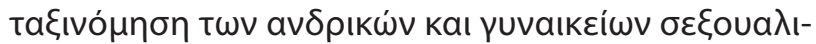

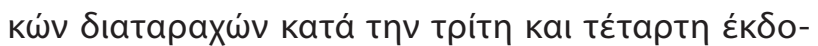

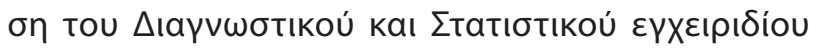

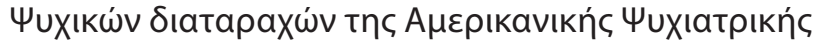

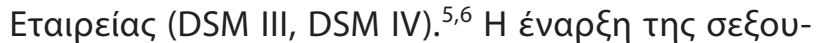

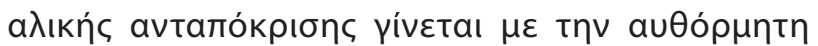

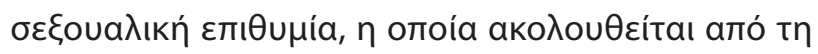

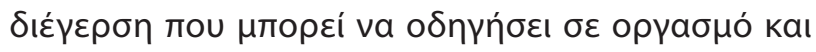

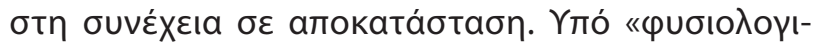

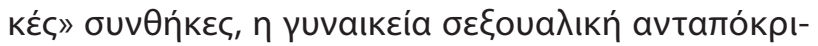

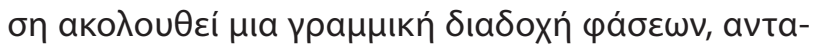

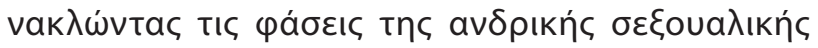
avtamókpions.

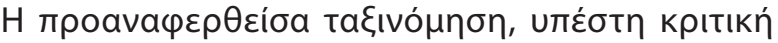

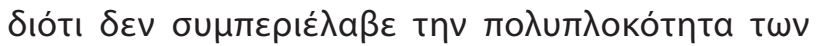

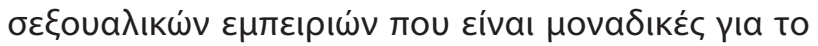

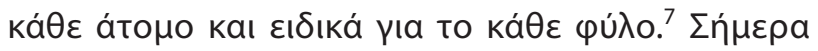

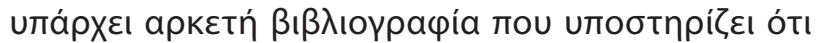

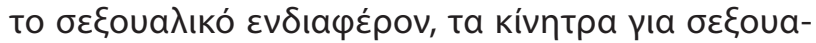




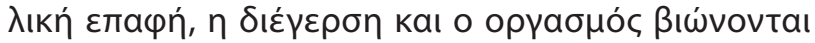

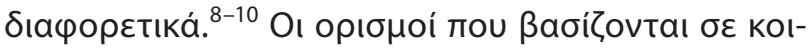

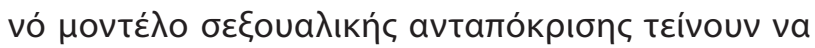

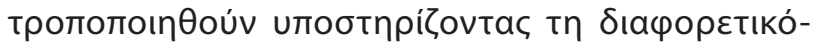

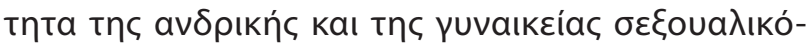

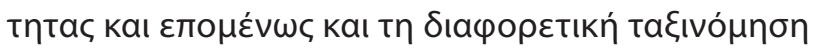

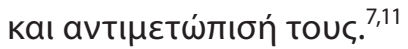

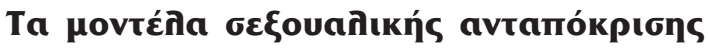

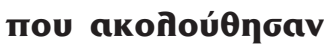

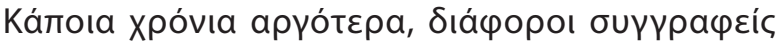

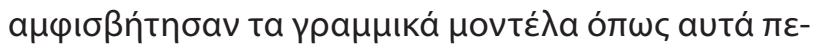
рıүра́чпкаv атó тоuc Masters \& Johnson \& Kaplan.

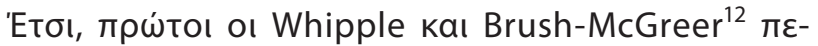

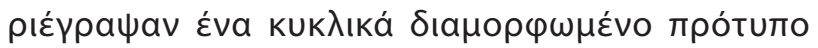

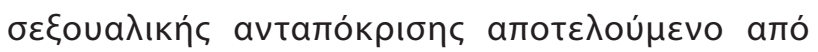

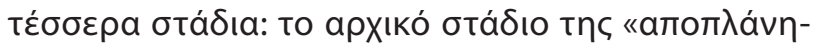

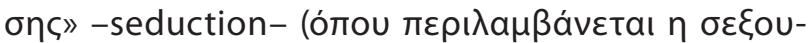

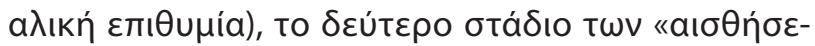

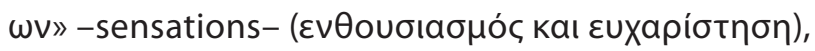

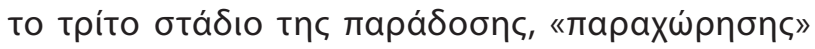

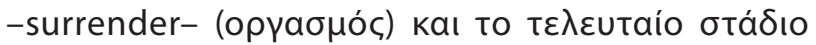

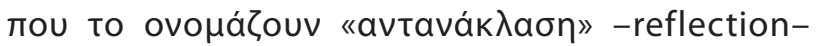

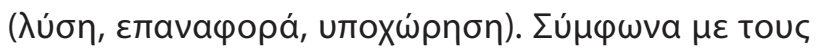

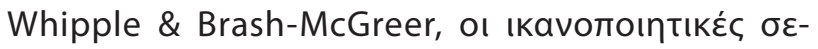

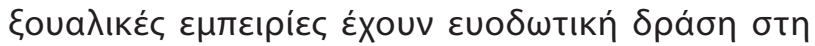

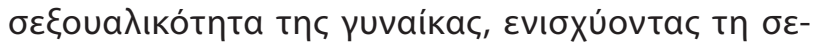

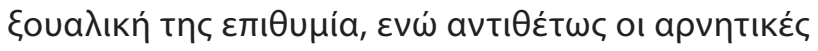

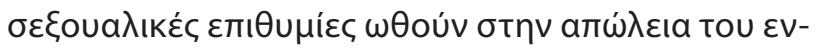

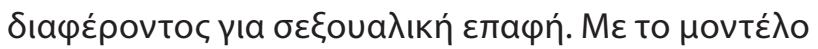

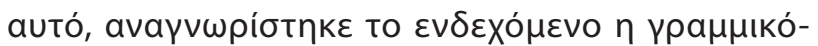

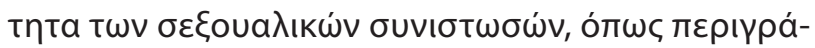
фпкаv ато́ тоuс Masters \& Johnson \& Kaplan, va

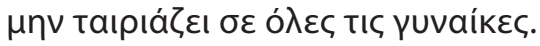

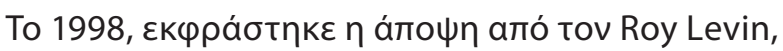

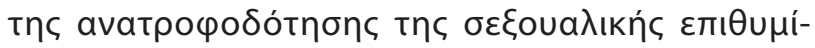

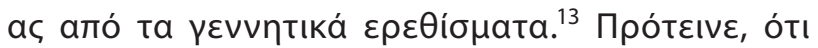

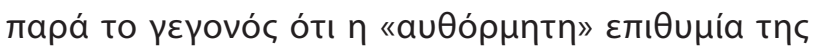

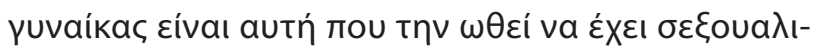

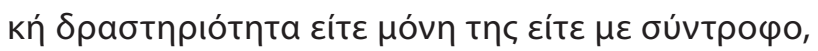

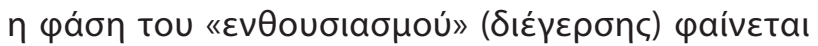

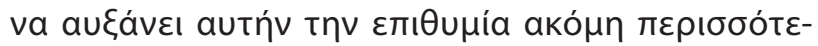

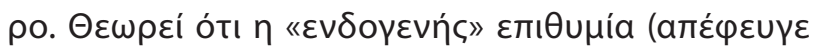

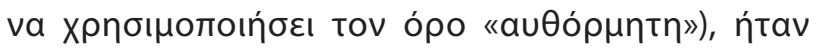

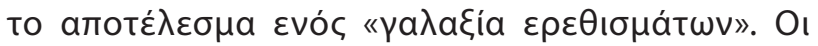

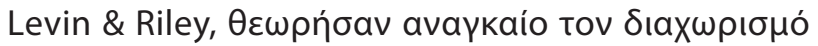

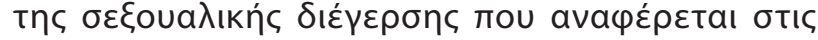

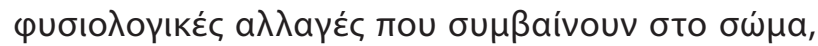

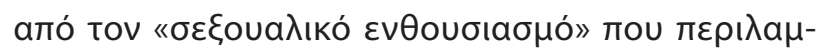

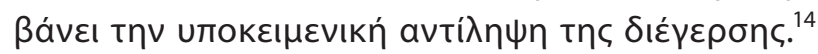

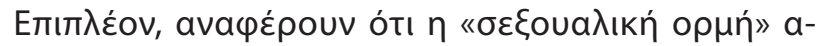

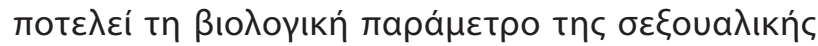

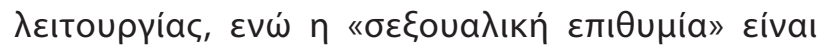

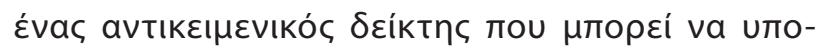

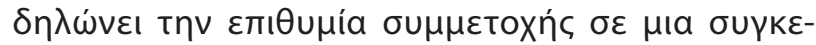

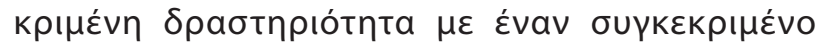

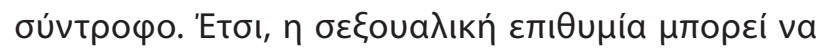

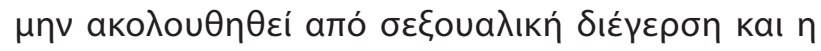

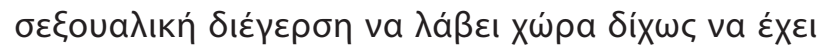

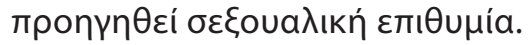

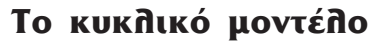 каı то DSM-5}

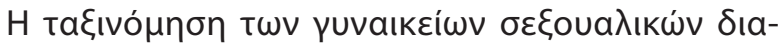

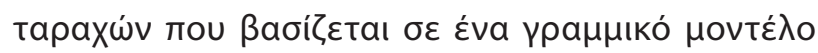

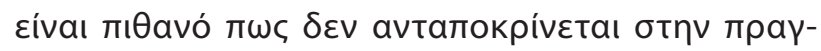

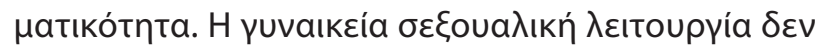

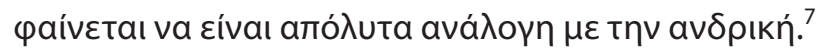

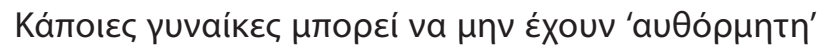

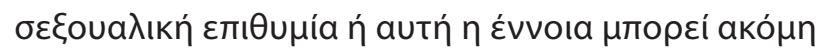
кaı va $\mu \eta v$ uтápxहı. ${ }^{15,16}$

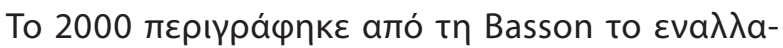

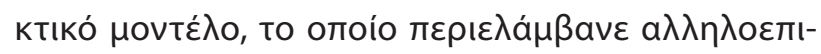

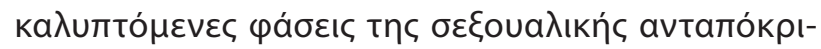

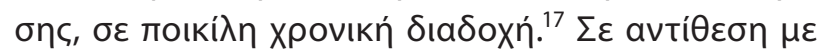

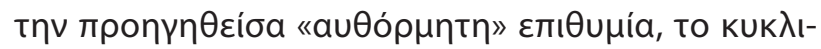

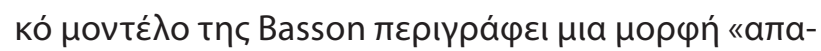

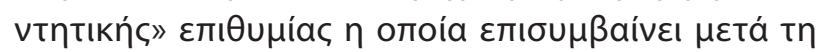

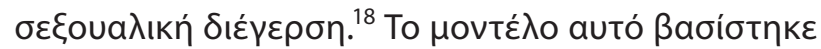

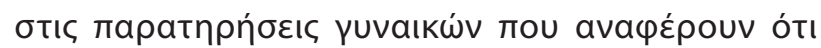

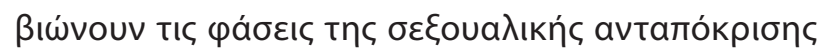

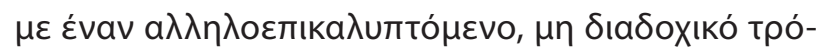

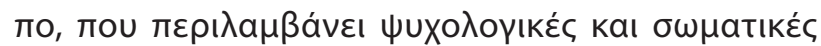

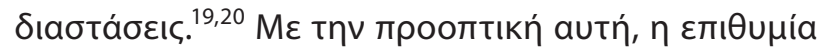

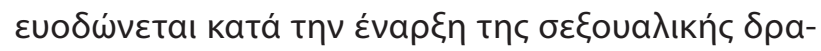

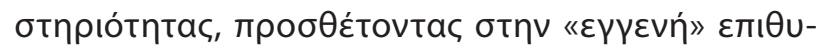

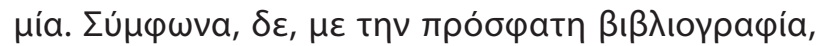

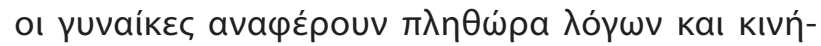

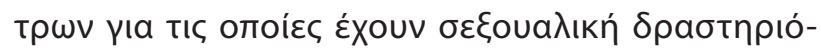

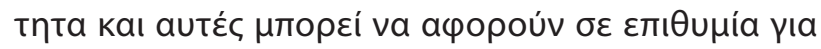

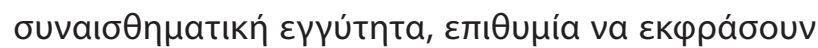

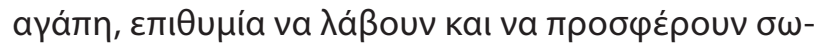




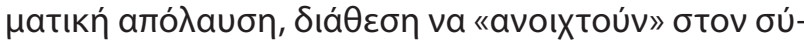

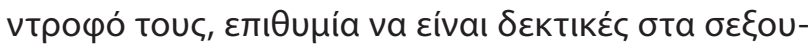

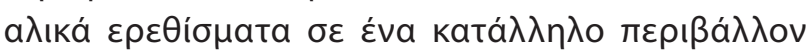

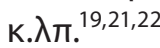

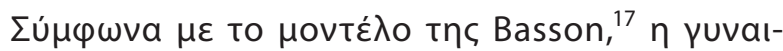

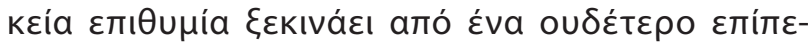

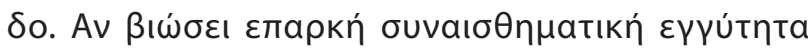

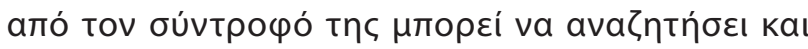

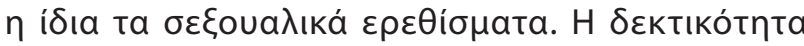

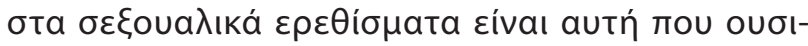

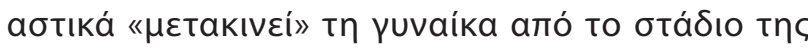

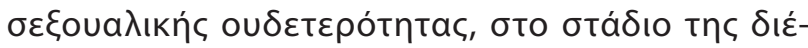

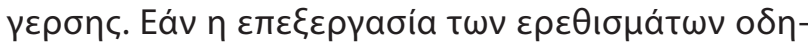

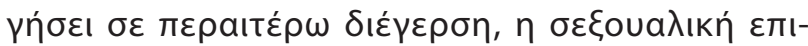

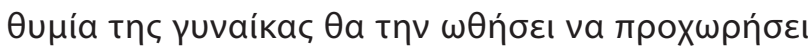

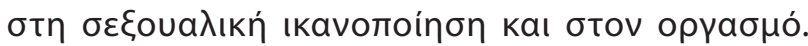

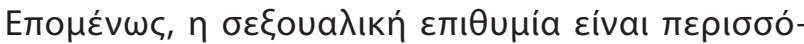

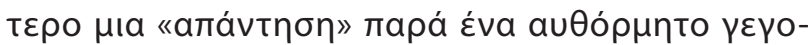

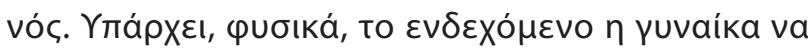

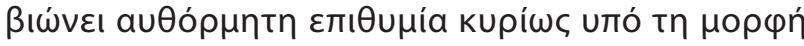

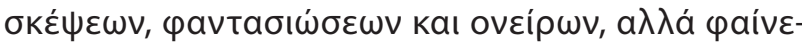

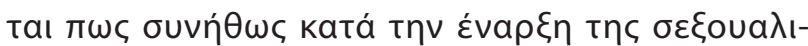

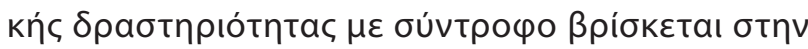

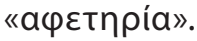

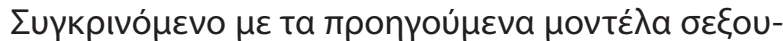

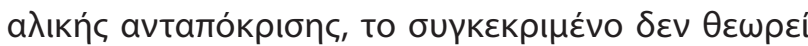

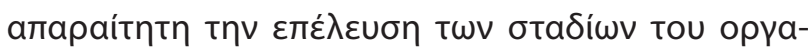

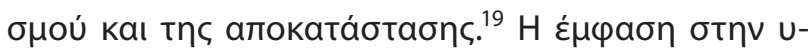

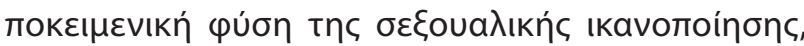

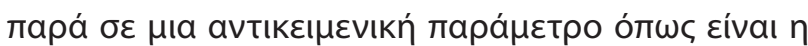

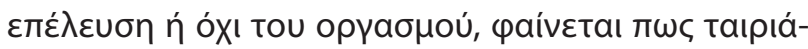

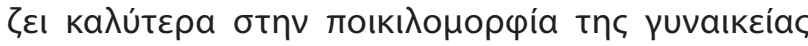

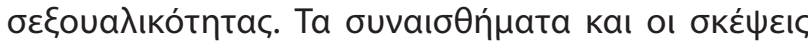

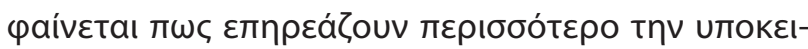

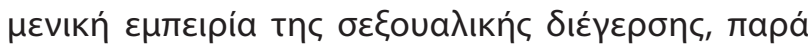

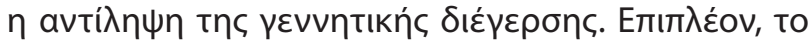

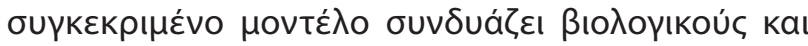

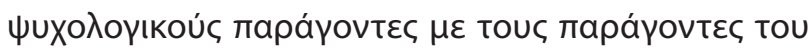

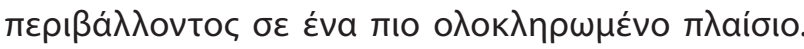

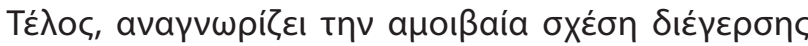

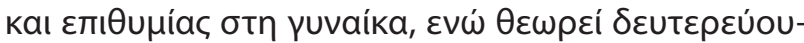

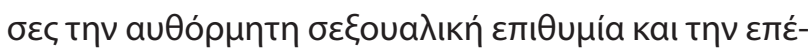
$\lambda \varepsilon$

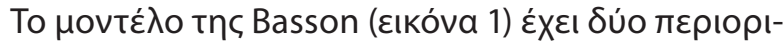

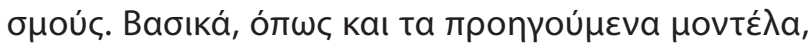

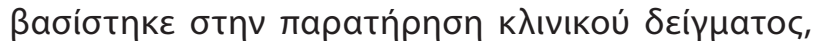

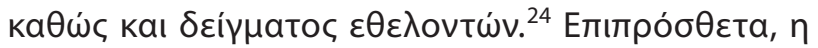

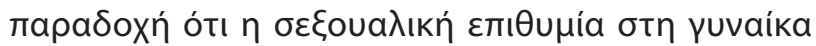

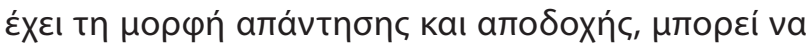

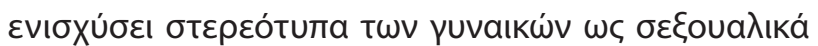

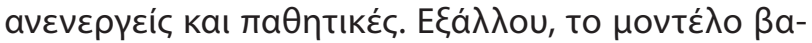

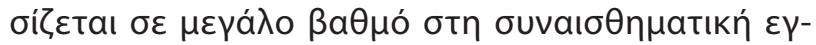

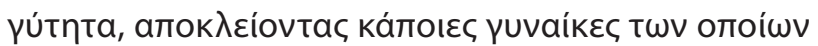

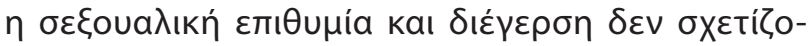

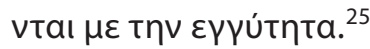

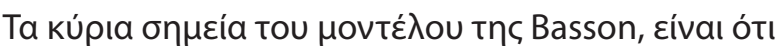

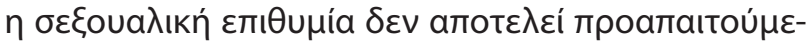

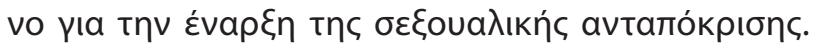

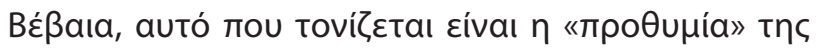

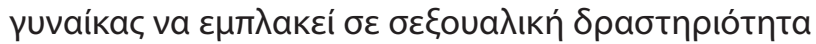

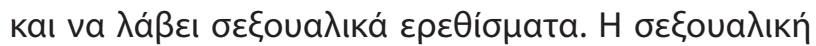

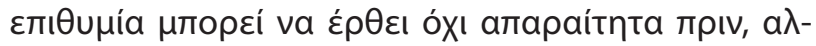

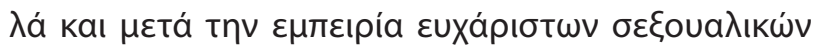

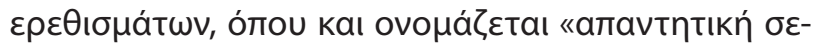

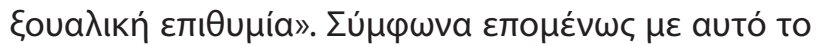

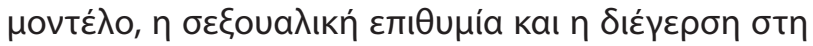

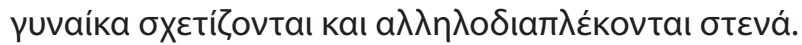

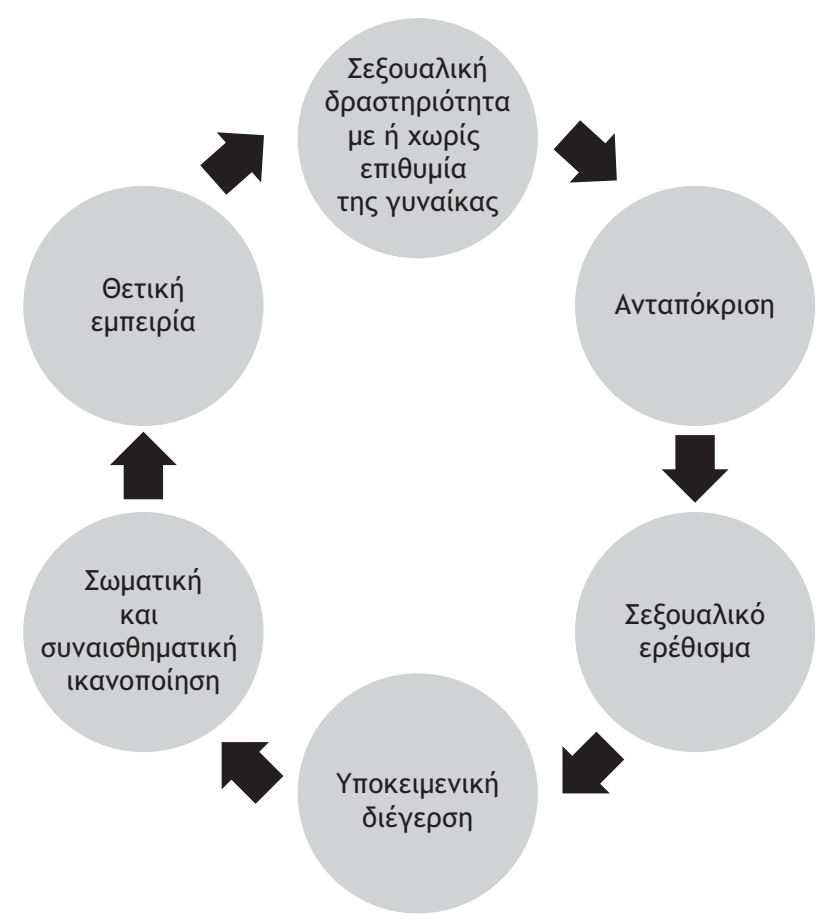

Eıkóva 1. Movtédo Basson. 


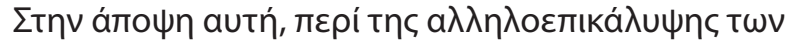

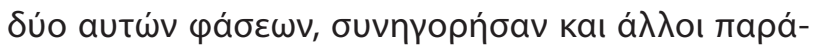

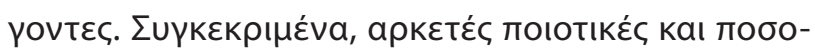

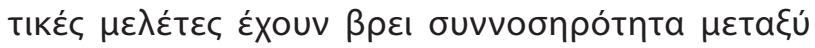

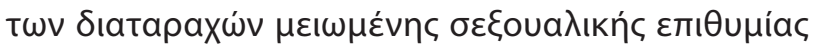

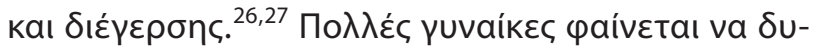

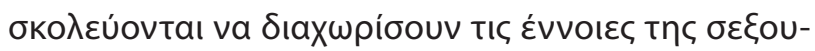

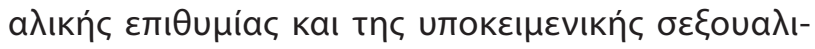

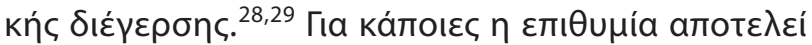

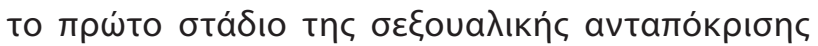

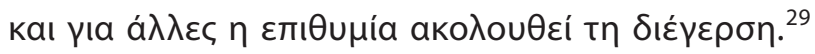

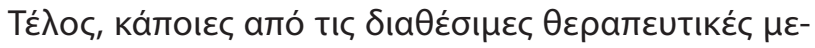

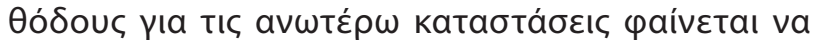

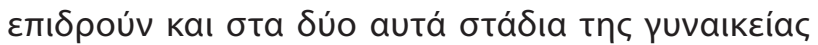

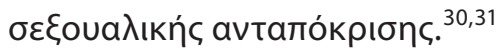

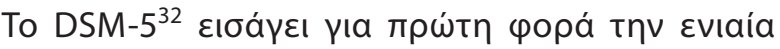

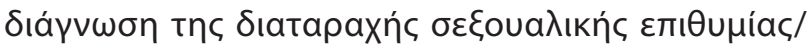

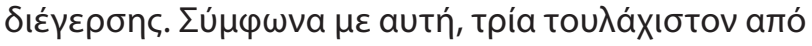

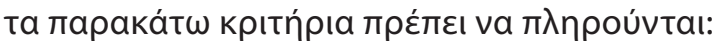

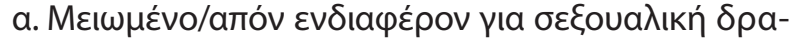
бтпріótпта.

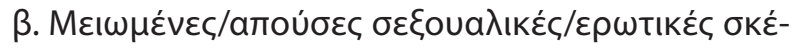

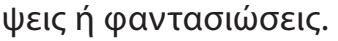

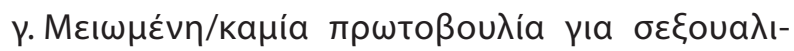

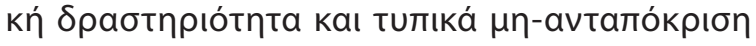

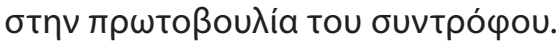

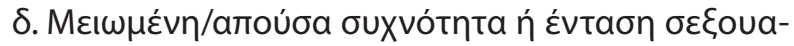

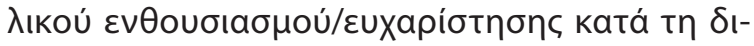

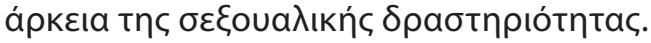

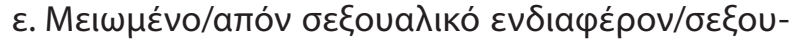

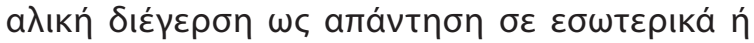

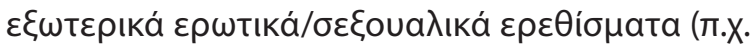

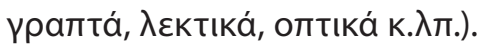

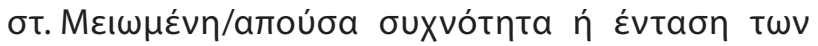

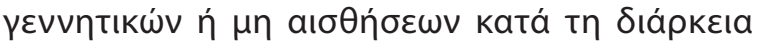

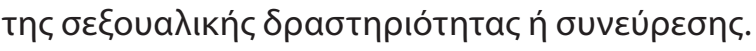

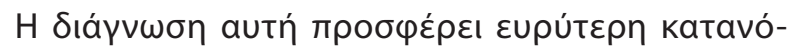

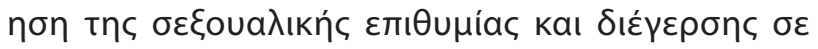

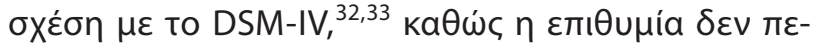

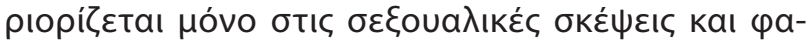

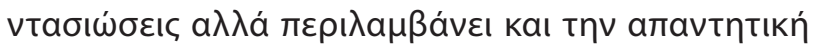

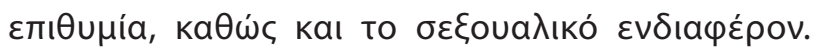

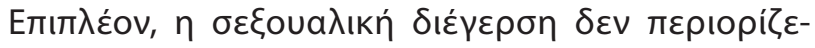

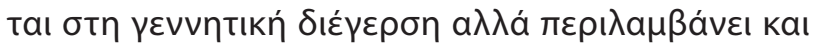

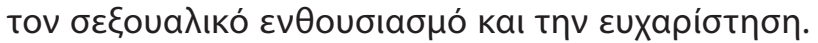

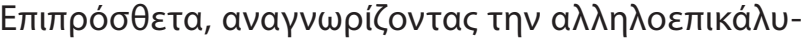

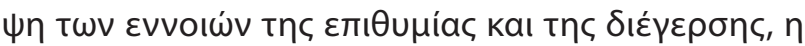

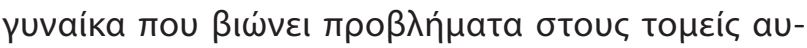

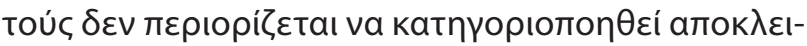

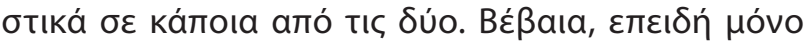

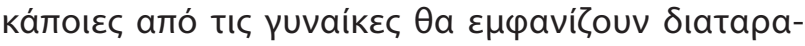

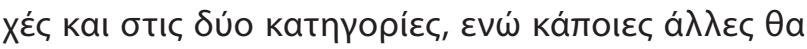

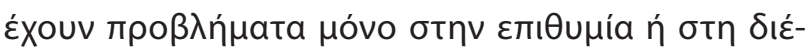

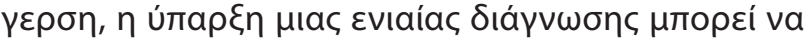

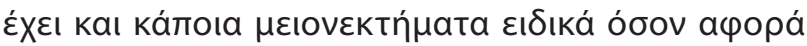

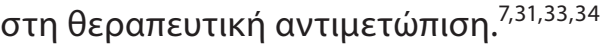

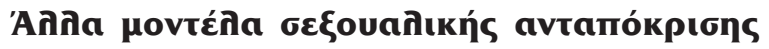

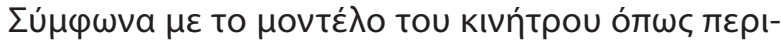

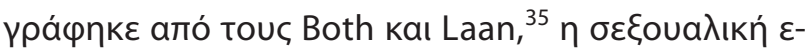

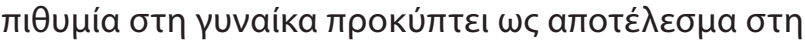

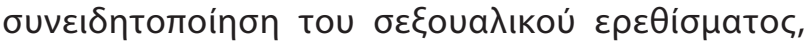

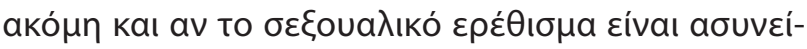

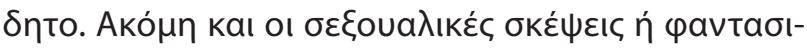

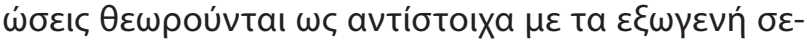

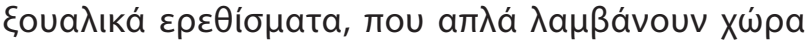

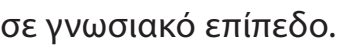

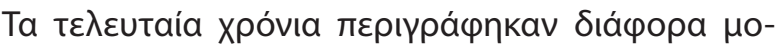

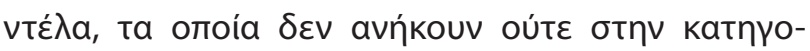

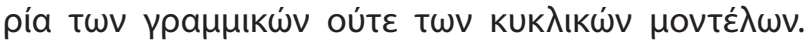

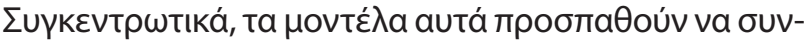

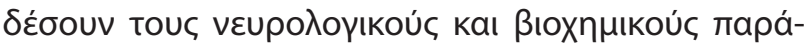

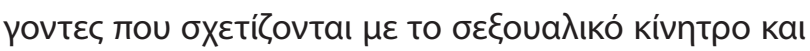

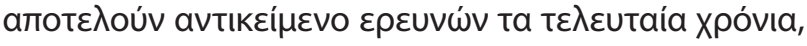

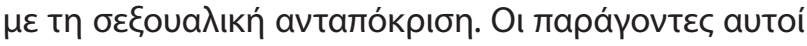

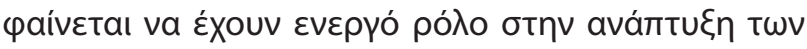

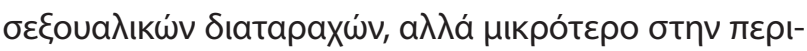

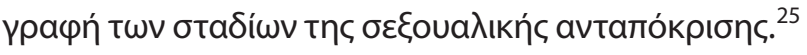

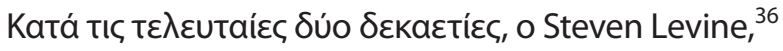

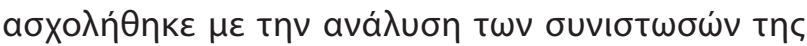

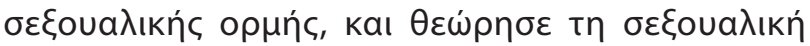

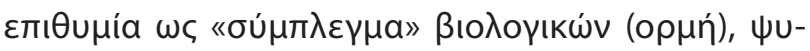

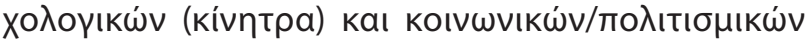

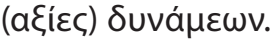

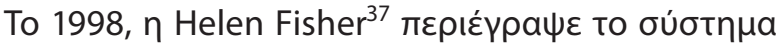

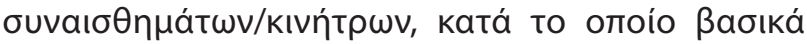

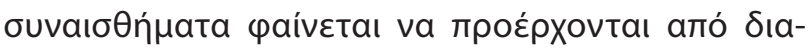

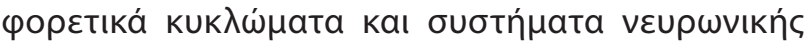

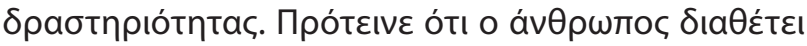

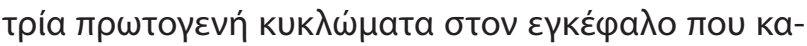




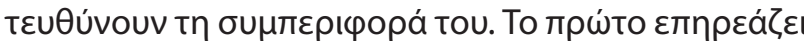

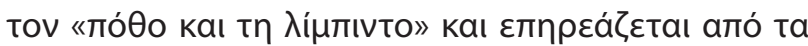

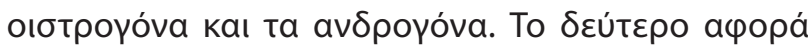

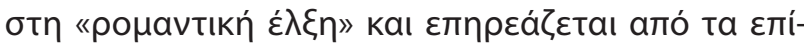

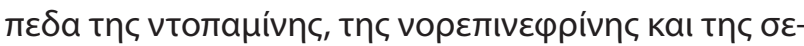

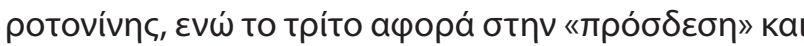

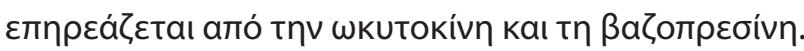

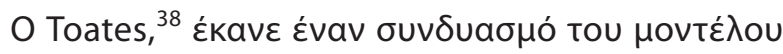

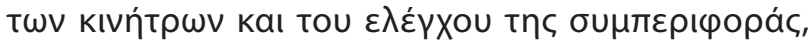

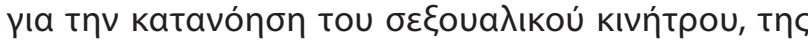

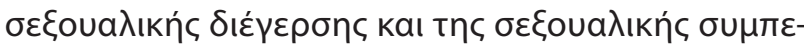

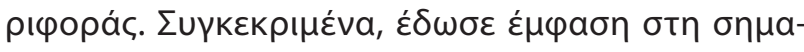

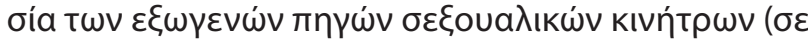

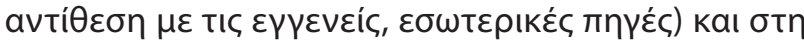

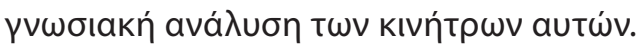

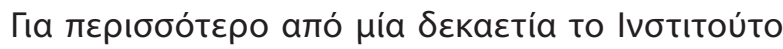

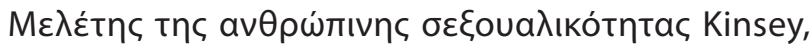

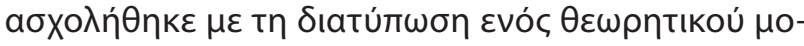

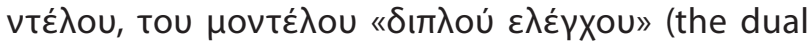

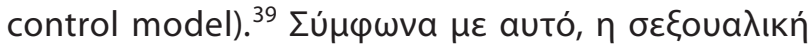

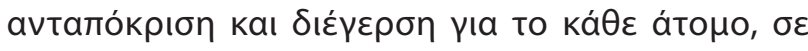

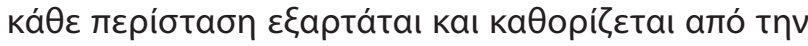

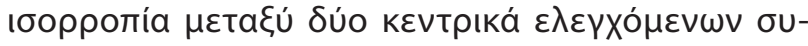

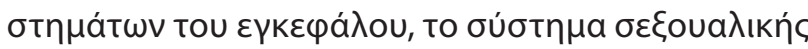

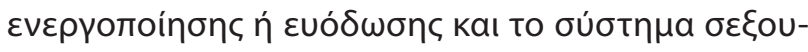

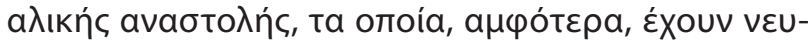
роßıооріко́ ито́ßаӨро. ${ }^{40}$

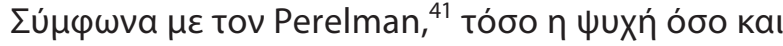

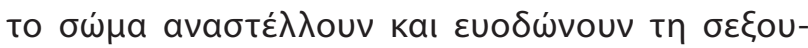

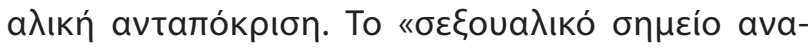

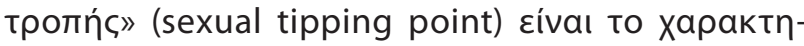

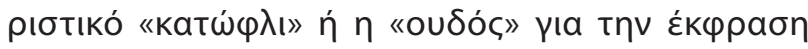

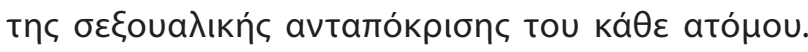

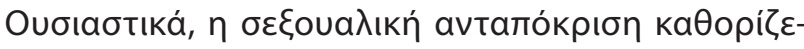

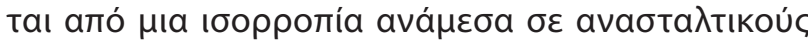

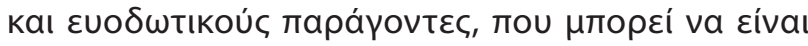

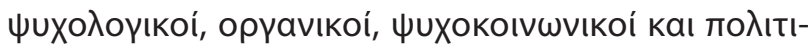

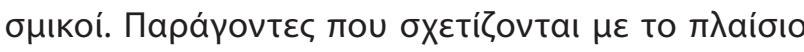

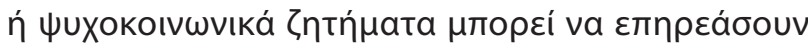
тпv avтапо́крібп.

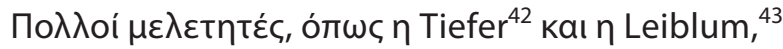

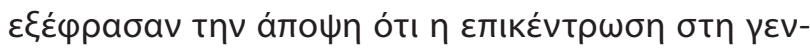

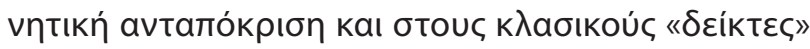

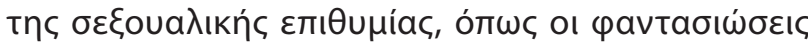

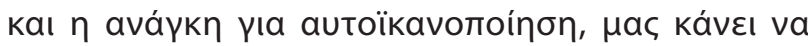

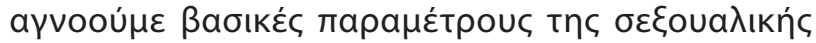

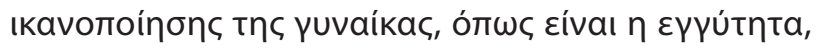

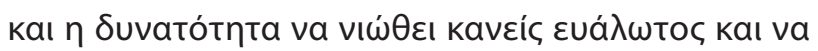

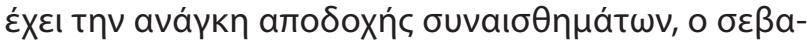

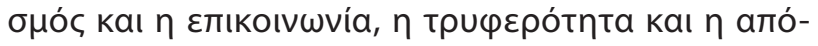

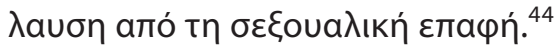

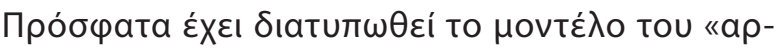

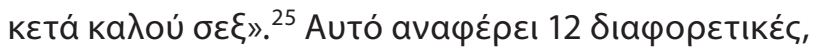

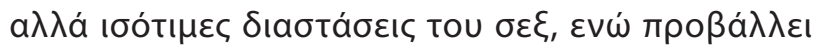

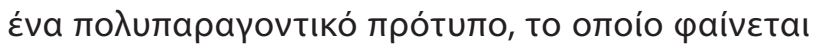

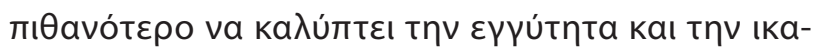

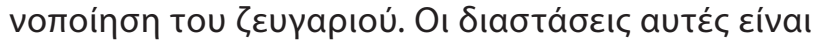

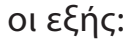

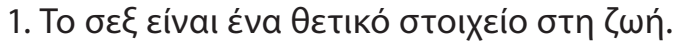

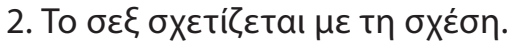

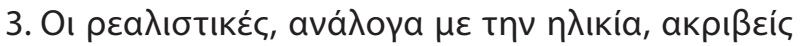

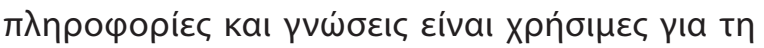

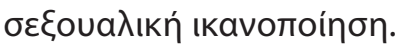

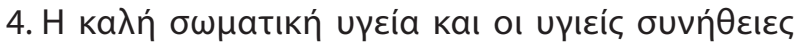

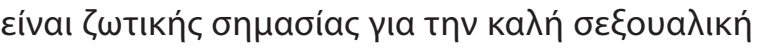
uүeía.

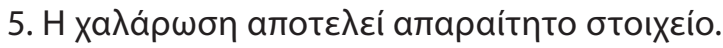

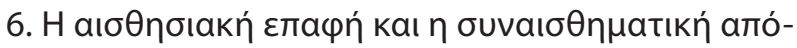

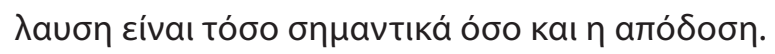

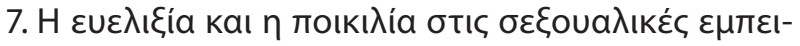

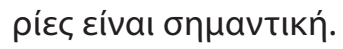

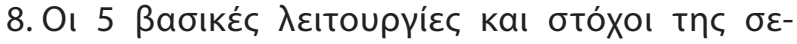

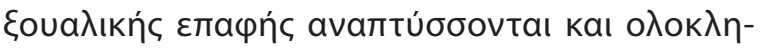

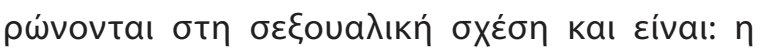

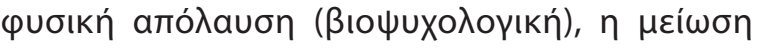

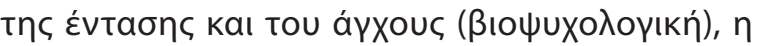

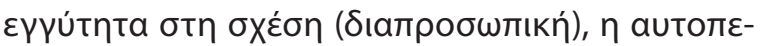

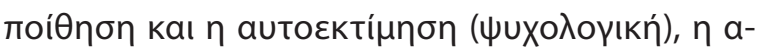

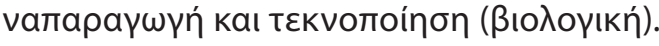

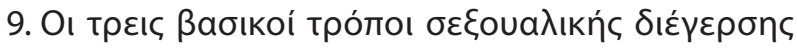

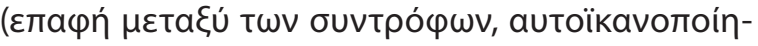

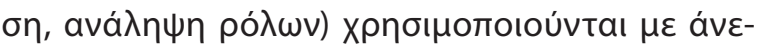

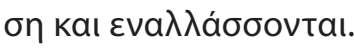

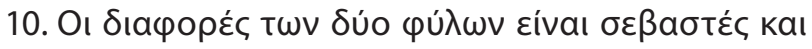

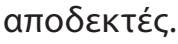

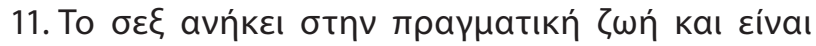

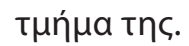




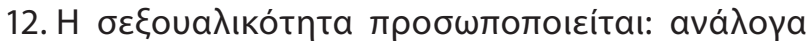

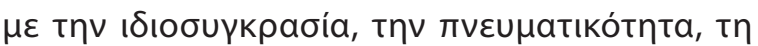

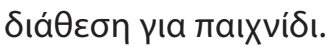

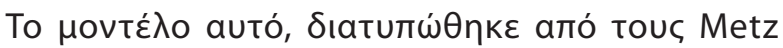

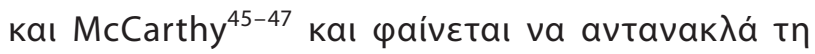

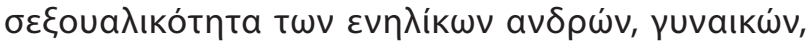

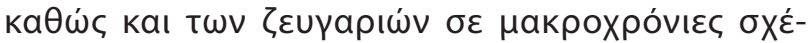

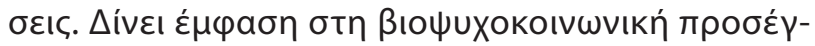

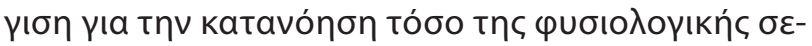

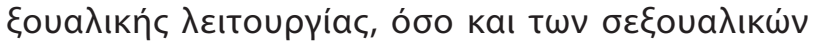

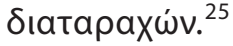

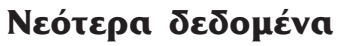

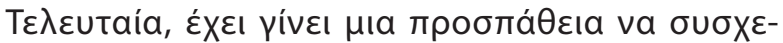

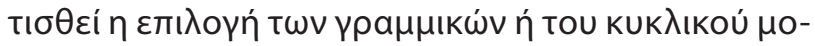

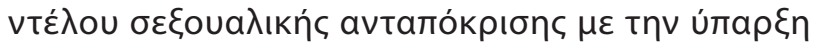

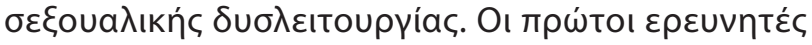

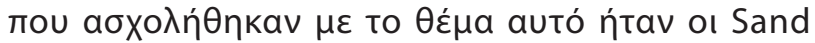

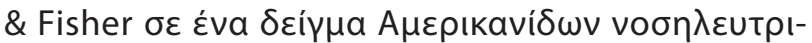

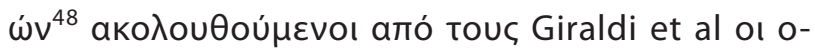

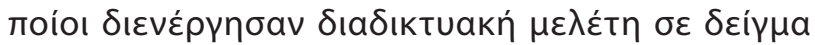
$\pi \lambda \eta \theta$

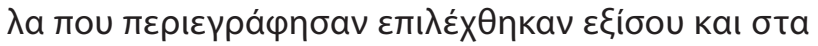

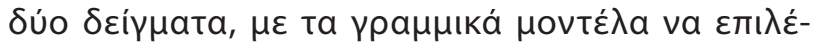

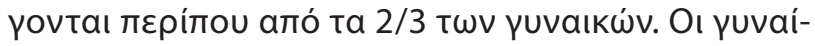

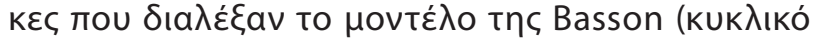

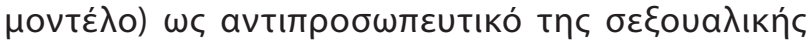

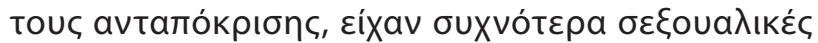

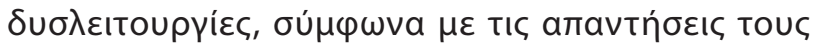
бто FSFI (Female Sexual Function Index). 'Ектотє,

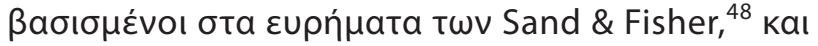

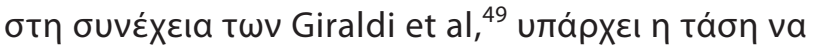

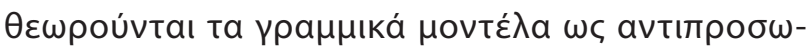

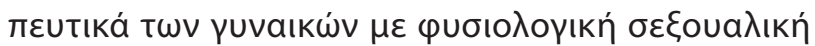

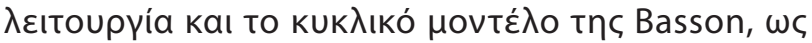

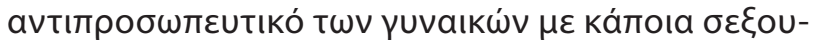

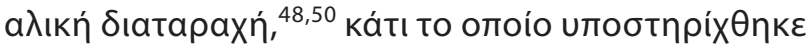

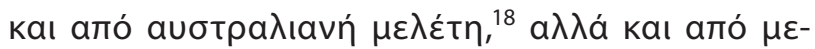

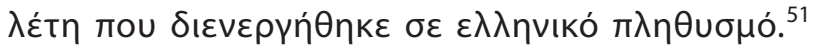

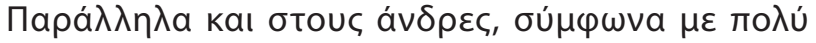

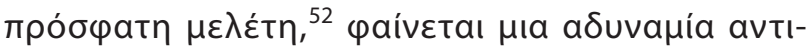

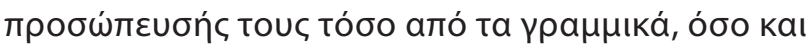

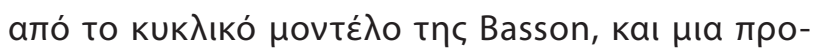

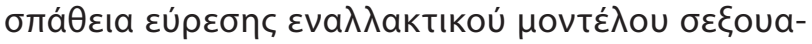

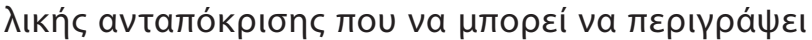

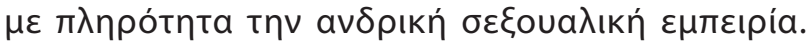

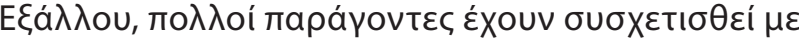

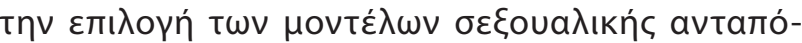

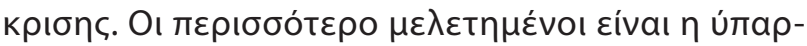

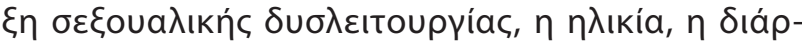

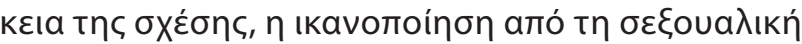

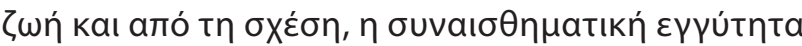

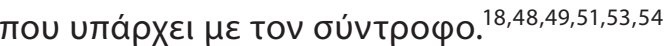

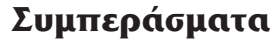

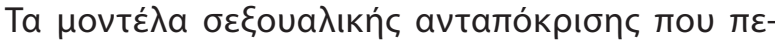

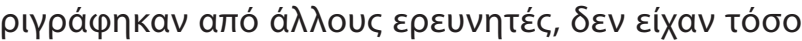

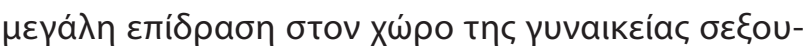

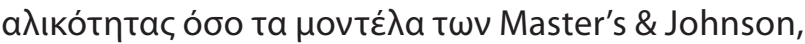

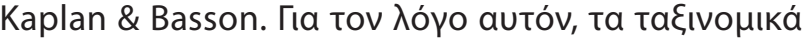

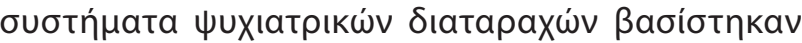

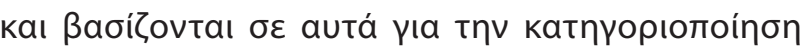

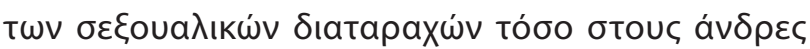

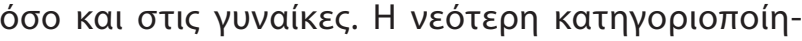

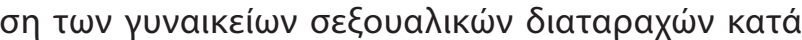

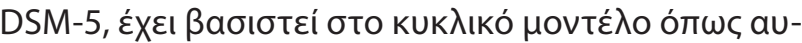

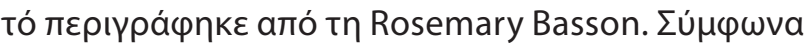

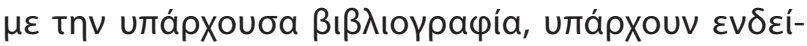

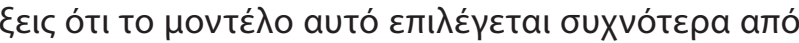

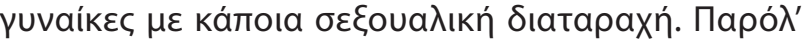

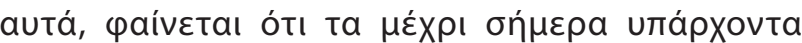

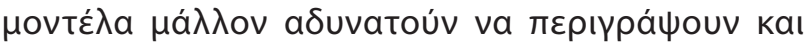

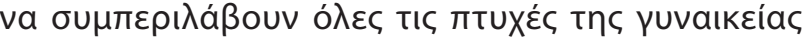

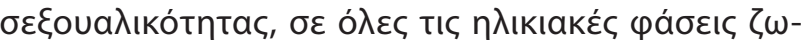

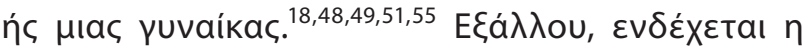

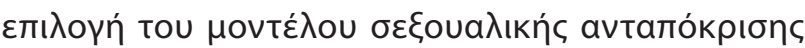

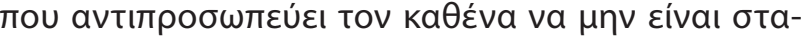

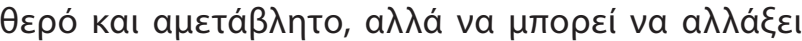

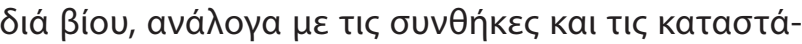

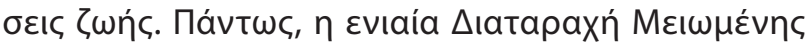

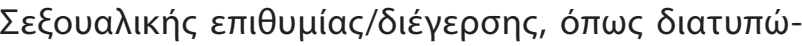

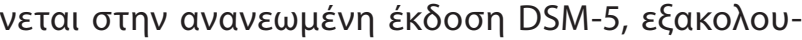

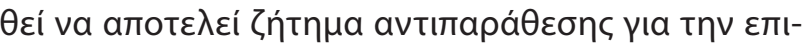

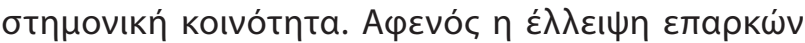

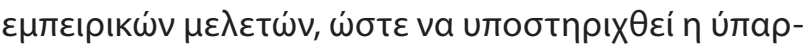

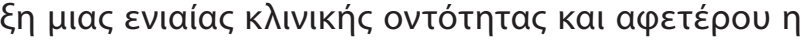

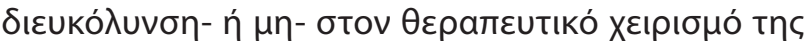

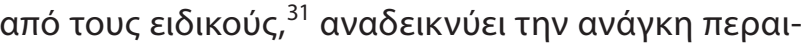

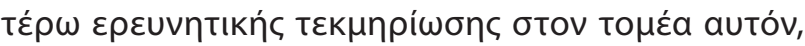

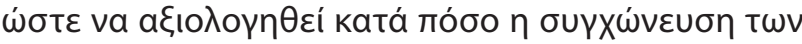

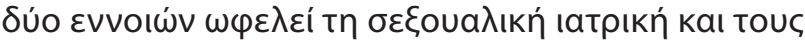

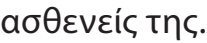




\title{
Women's sexual function through sexual response models: From DSM-IV to DSM-5
}

\author{
F. Ferenidou, ${ }^{1,2}$ P.S. Kirana, ${ }^{2}$ L. Athanasiadis ${ }^{1}$ \\ ${ }^{1} 1 s t$ Psychiatric Clinic of the Aristotle University of Thessaloniki, \\ ${ }^{2}$ Institute for the Study of Urologic Diseases, Aristotle University of Thessaloniki, Thessaloniki, Greece
}

Psychiatriki 2017, 28:322-331

Until recently, DSM classifications regarding women's sexual dysfunctions were based on definitions that mainly derived from experts opinions, rather than clinical or epidemiological studies. Additionally, this classification was the same for men and women, taking for granted the existence of a single sexual response model for men and women. More specifically, the main models that were described and prevailed were the linear models of Master's \& Johnson and Kaplan, as well as the circular model of Rosemary Basson that followed. The differences between linear and circular models have to do mainly with the fact that stages of sexual response overlap with each other in the circular model, while the initiation of sexual activity may not need the presence of spontaneous sexual desire. In linear models, the stages of sexual desire, arousal, orgasm and resolution follow each other in a stable manner, in contrary to circular models where sexual desire may not be neither the first stage nor the main reason for sexual activity. In this case, reasons for sexual activity may vary, focusing also on other aspects of human sexuality and personality and not only on innate sexual desire. DSM-5 based the classification of sexual dysfunctions on the circular model, although there is still not enough evidence to prove that all men and women can be represented by the same sexual response model throughout the lifespan. Apart from the aforementioned sexual response models, also other models have been described by several investigators, making an important effort to give a more accurate and complete description of human sexual function, as well as its disorders. The present article makes an attempt to review the sexual response models that have been described by the literature up to date, as well as to relate them to the DSM-IV and DSM-5 classifications.

Key words: Sexual response models, female sexual dysfunctions, DSM-IV, DSM-5.

\section{BıßAtoypacpía}

1. Masters W, Johnson V. Human sexual response. Boston, Little, Brown \& Co, 1966

2. Kaplan H. The new sex therapy. New York, Brunner, Mazel, 1974

3. DeRogatis L, Clayton AH, Rosen RC, Sand M, Pyke RE. Should sexual desire and arousal disorders in women be merged? Arch Sex behav 2011, 40:217-219, doi: 10.1007/s10508-010-9677-1

4. Brotto L, Graham CA, Binik YM, Segraves TR, Zucker KJ. Should sexual desire and arousal dirorders in women be merged? A response to DeRogatis, Clayton, Rosen, Sand and Pyke. Arch Sex Behav 2010, 40:221-225, doi 10.1007/s10508-010-9706-0
5. American Psychiatric Association. Diagnostic and statistical manual of mental disorders. 3rd ed. Text revision. American Psychiatric Association, Washington DC, 1980

6. American Psychiatric Association. Diagnostic and statistical manual of mental disorders. 4th ed. Text revision. American Psychiatric Association, Washington DC, 2000

7. Sungur GA. Critiques and challenges to old and recently proposed American Psychiatric Association's website DSM 5 diagnostic criteria for sexual dysfunctions. Klin Psikofarmakol B 2013, 23:113-128, doi: 10.5455/bcp.20130416063859 
8. Chivers BJ. A sex difference in features that elicit genital response. Biol Psychol 2005, 70:115-120, doi: 10.1016/j. biopsycho.2004.12.002

9. Chivers TA. Effects of gender and relationship context in audio narratives on genital and subjective sexual response in heterosexual women and men. Arch Sex Behav 2012, 41:185-197, doi: 10.1007/s10508-012-9937-3

10. Laan W, van Bellen G, Hanewald G. Women's sexual and emotional responses to male- and female- produced erotica. Arch Sex Behav 1994, 23:153-169, PMID:7517135

11. Leiblum S. Sex Therapy Today Current Issues and Future Perpectives. In: Leiblum S (ed) Principles and practice of sextherapy. Guilford Press, New York, NY, 2007:3-22

12. Whipple B, Brash-McGreer KB. Management of female sexual dysfunction. In: Sipski MLAC (ed) Sexual function in people with disability and chronic illness. A health professional's guide. Gaithersburg, Aspen Publishers, 1997:509-534

13. Levin R. Sexual desire and the deconstruction and reconstruction of the human female sexual response model of Masters and Johnson. In: Everaerd W, Laan E, Both S (eds) Sexual appetite, desire and motivation: energetics of the sexual system. Proceedings of the colloquium. Academy of Arts and Sciences, Amsterdam: Royal Netherlands, 1998

14. Levin R, Riley A. Sexual disorder and psychosexual therapy. Psychiatry 2007, 6:90-94

15. Laan EvDE, van Lunsen RH. Genital responsiveness in healthy women with and without sexual arousal disorder. J Sex Med 2008, 5:1424-1435, doi: 10.1111/j.1743-6109.2008.00827.x

16. Laan E, Both $\mathrm{S}$. What makes women experience desire? Fem Psychol 2008, 18:505-514, doi: 10.1177/0959353508095533

17. Basson R. The female sexual response: A different model. J Sex Marital Ther 2000, 26:51-65, doi: 10.1080/009262300278641

18. Giles K, McCabe MP. Conceptualizing women's sexual function: linear vs. circular models of sexual response. J Sex Med 2009, 6:2761-271, doi: 10.1111/j.1743-6109.2009.01425.x

19. Basson R. Women's sexual dysfunction: revised and expanded definitions. CMAJ 2005, 172:1327-1333, doi: 10.1503/ cmaj.1020174

20. Dennerstein L, Lehert P. Modeling mid-aged women's sexual functioning: a prospective, population-based study. J Sex Marital Ther 2004, 30:173-183, doi: 10.1080/00926230490262375

21. Basson R, Wierman ME, van Lankveld J, Brotto L. Summary of the recommendation on sexual dysfunction in women. J Sex Med 2010, 7:314-326, doi: 10.1111/j.1743-6109.2009.01617.x

22. Meston CM, Buss DM. Why humans have sex. Arch Sex Behav 2007, 36:477-507, doi: 10.1007/s10508-007-9175-2

23. Rosen RC, Barsky JL. Normal sexual response in women. Obstet Gynecol Clin North Am 2006, 33:515-526, doi: 10.1016/j.ogc.2006.09.005

24. Wylie K, Mimoun S. Sexual response models in women. Maturitas 2009, 63:112-115, doi: 10.1016/j.maturitas. 2009.03.007

25. Tripodi SC, Simonelli C. Psychology of sexual response. In: Porst HRY (ed) The ESSM Syllabus of sexual medicine. European Society for Sexual Medicine, MEDIX, Milano, 2012: $83-141$
26. Bozman AW, Beck JG. Covariation of sexual desire and sexual arousal: The effects of anger and anxiety. Arch Sex Behav 1991, 20:47-60, PMID: 2003771

27. Sanders S, Graham CA, Milhausen RR. Predicting sexual problems in women: the relevance of sexual excitation and sexual inhibition. Arch Sex Behav 2008, 37:241-251, doi: 10.1007/ s10508-007-9235-7

28. Brotto L, Heiman JR, Tolman DL. Narratives of desire in midage women with and without arousal difficulties. J Sex Res 2009, 46:387-398, doi: 10.1080/00224490902792624

29. Graham C, Sanders SA, Milhausen RR, McBride KR. Turning on and turning off: a focus group study of the factors that affect women's sexual arousal. Arch Sex Behav 2004, 33:527-538, doi: 10.1023/B:ASEB.0000044737.62561.fd

30. Hurlbert DF. A comparative study using orgasm consistency training in the treatment of women reporting hypoactive sexual desire. Arch Sex Behav 1993, 19:49-55, doi: 10.1080/00926239308404887

31. Kirana P. Female Sexual Arousal Disorders. In: Kirana PSTF Reisman $\mathrm{Y}$, Porst $\mathrm{H}$ (eds) The EFS and ESSAM syllabus of clinical sexology. MEDIX, Amsterdam, 2013:786-801

32. American Psychiatric Association. Diagnostic and statistical manual of mental disorders. 5th ed. American Psychiatric Publishing, Arlington, VA, 2013

33. Derogatis LE, Brauer M, van Lunsen RH, Jannini EA, Davis SR et al. Responses to the proposed DSM-V changes. J Sex Med 2010, 7:1998-2014, doi: 10.1111/j.1743-6109.2010.01865.x

34. Sungur GA. A comparison of DSM-IV-TR and DSM-V definitions for sexual dysfunctions: critiques and challenges. J Sex Med 2014, 11:364-373, doi: 10.1111/jsm.12379

35. Both S, Everaerd W, Laan E. Desire emerges from excitement: a psychophysiological perspective on sexual motivation. In: Janssen E (ed) The psychophysiology of sex. Indiana University Press, Bloomington, 2007:327-339

36. Levine S. Demystifying love. Routledge, New York, 2008

37. Fisher $\mathrm{H}$. Lust, attraction, and attachment in mammalian reproduction. Hum Nat 1998, 9:23-52, doi: 10.1007/s12110-998-10105

38. Toates F. An integrative theoretical framework for understanding sexual motivation, arousal, and behavior. J Sex Res 2009, 46:168-193, doi: 10.1080/00224490902747768

39. Bancroft J, Janssen E. The dual control model of male sexual response: a theoretical approach to centrally-mediated erectile dysfunction. Neurosci Biobehav Rev 2000, 24:571-579, PMID: 10880822

40. Bancroft J. Central inhibition of sexual response in the male: a theoretical perspective. Neurosci Biobehav Rev 1999, 23:763784, PMID: 10541055

41. Perelman M. The Sexual Tipping Point: a mind/body model for sexual medicine. J Sex Med 2009, 6:629-632, doi: 10.1111/j.1743-6109.2008.01177.x

42. Tiefer L. Historical, scientific, clinical and feminist criticisms of "the human sexual response cycle". Annu Rev Sex Res 1991, $2: 1-23$

43. Leiblum S. Definitions and classification of female sexual disorders. Int J Impot Res 1998, 10:104-106, PMID:9647970 
44. Babar K, Murray $\mathrm{Cl}$. A postmodern feminist approach to teaching human sexuality. Fan Relay 2001, 50:23-33, doi: 10.1111/ j.1741-3729.2001.00023.x

45. McCarthy MM. The "Good-Enough Sex" model: a case illustration. Sex Relat Ther 2008, 23:227-234, doi: 10.1080/1468 1990802165919

46. Metz M, McCarthy B. The Good-Enough Sex model for couple satisfaction. Sex Relat Ther 2007, 22:351-362, doi: 10.1080/ 14681990601013492

47. Metz M, McCarthy B. Enduring desire: Your guide to lifelong intimacy. Routledge, New York, 2010

48. Sand M, Fisher WA. Women's endorsement of models of female sexual response: the nurses' sexuality study. J Sex Med 2007, 4:708-719, doi: 10.1111/j.1743-6109.2007.00496.x

49. Giraldi A, Kristensen EMS. Endorsement of models describing sexual response of men and women with a sexual partner: An online survey in a population sample of Danish adults ages 20-65 years. J Sex Med 2015, 12:116-128, doi: 10.1111/ jsm. 12720

50. Meana M. Elucidating women's (hetero)sexual desire: definitional challenges and content expansion. J Sex Res 2010, 47:104-122, doi: 10.1080/00224490903402546

51. Ferenidou F, Kirana PS, Fokas K, Hatzichristou D, Athanasiadis L. Sexual response models: Toward a more flexible pattern of women's sexuality. J Sex Med 2016, 13:1369-1376

52. Connaughton C, Karantzas G. Conceptualization of the sexual response models in men: Are there differences between sexually functional and dysfunctional men? J Sex Med 2016, 13:453-463, doi: 10.1016/j.jsxm.2015.12.032

53. McCall $\mathrm{K}$, Meston, C. Cues resulting in desire for sexual activity in women. J Sex Med 2006, 3:838-852, doi: 10.1111/j.17436109.2006.00301.x

54. Balon R. The DSM criteria of sexual dysfunction: Need for a change. J Sex Marital Ther 2008, 34:186-197, doi: 10.1080/ 00926230701866067

55. Meston C, Hamilton LD, Harte CB. Sexual motivation in women as a function of age. $J$ Sex Med 2009, 6:3305-3319, doi: 10.1111/j.1743-6109.2009.01489.x

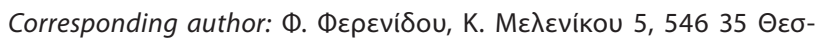
ба入ovíkn e-mail: fferenidou@yahoo.com 